\title{
ON G-OPEN SET
}

\section{Jalal Hatem Hussein*}

Date of acceptance 9/5/2007

\begin{abstract}
:
\section{Introduction}

In recent years a number of generalizations of open sets have been considered. Seven of these notions were defined similarly using closure operator (cl) and the interior (int) in the following way.
\end{abstract}

A new class of generalized open sets in a topological space, called $G$-open sets, is introduced and studied. This class contains all semi-open, preopen, b-open and semipreopen sets. It is proved that the topology generated by G-open sets contains the topology generated by preopen,b-open and semi-preopen sets respectively.

DEFINITION 1. A subset $S$ of a space $(X, T)$ is called:

(1) $\alpha$-set if $S \subset$ int(cl(intS)),

(2)Semi-open ifS $\subset_{\mathrm{cl}(\mathrm{int} S) \text {, }}$

(3)Preopen if $S \subset$ int(clS),

(4)Regular open if $\mathrm{S}=\operatorname{int}(\mathrm{clS})$,

(5)Semi-regular if and only if there exists a regular open set $u$ with $\mathrm{u} \subset_{\mathrm{S}} \subset_{\text {clu, }}$

(6)b-open if $S \subset_{\mathrm{cl}(\text { intS })} \cup_{\text {int }}(\mathrm{clS})$,

(7)Semi-preopen if $\mathrm{S} \subset \operatorname{cl}(\operatorname{int}(\mathrm{cl} S)$.

The first three notions are due to Njastad [10], Levine [1] and Mashhour [11], respectively. The concept of a preopen set was introduced by Corson and Michael [2] who used the term "locally dense". D. Andrijevic was introduce the concept of b-open and semi-preopen in [9], [3] respectively. We denote the classes of $\alpha$-set semiopen, preopen, b-open and semi-preopen sets in a space $(X, T)$ by $\mathrm{T} \alpha, \mathrm{SO}(\mathrm{X})$, $\mathrm{PO}(\mathrm{X}), \mathrm{BO}(\mathrm{X})$ and $\mathrm{SPO}(\mathrm{X})$ respectively. All of them are larger than $\mathrm{T}$ and closed under forming arbitrary unions. Njastad [10] showed that $T \alpha$ is a topology on $X$. In general, $\mathrm{SO}(\mathrm{X})$ need not be a topology on $\mathrm{X}$, but the intersection of a semi-open set and an open set is semiopen. The same holds for $\mathrm{PO}(\mathrm{X}), \mathrm{BO}(\mathrm{X})$ and $\operatorname{SPO}(\mathrm{X})$ respectively. The complement of a semi-open set is called semi-closed. Thus $\mathrm{S}$ is semi-closed if and only if $\operatorname{int}(\mathrm{clS}) \subset_{\mathrm{S}}$. Preclosed set is similarly defined. For a subset $\mathrm{S}$ of a space $\mathrm{X}$ preclosure of $\mathrm{S}$, denoted by pclS is the intersection of all preclosed subsets of $\mathrm{X}$ containing $\mathrm{S}$. The preinterior (resp. semi-preinterior)of $\mathrm{S}$, denoted by pintS (resp., spintS) is the union of all preopen (resp. semipreopen) subsets of $\mathrm{X}$ contained in $\mathrm{S}$. The definitions(4),(5) was defined by, Maio and Noiri [5], D.S.Jankovic [4], respectively. $\mathrm{By} \mathrm{cl}_{\alpha}$ and int $_{\alpha}$ we denote the closure and the interior operator in $\left(\mathrm{X}, \mathrm{T}_{\alpha}\right)$.

An extensive study of these operators was done in [3]. We recollect some of the relations that, together with their duals, we shall use in the sequel.

PROPOSITION 1.1. Let $S$ be a subset of $(X, T)$. Then:

(1) $\operatorname{pcl} S=S \cup \operatorname{cl}($ int $S$ ),

(2) $\operatorname{pint} S=S \cap \operatorname{int}(\mathrm{cl} S)$,

(3) int $_{\alpha} S=S \cap \operatorname{int}(\operatorname{clint} S$ ),

(4) $\operatorname{spint} S=S \cap \operatorname{cl}(\operatorname{int}(\operatorname{cl} S)$,

(5) $\operatorname{pcl}(\operatorname{pint} S)=\operatorname{pint} S \cup \operatorname{cl}(\operatorname{int} S)$.

2. Some properties of $\mathbf{G}$ - open sets.

Now we consider a new class of generalized open sets.

DEFINITION 2. A subset $\mathrm{S}$ of a space $(\mathrm{X}, \mathrm{T})$ is called G-open if $\mathrm{S} \subset \mathrm{cl}(\operatorname{int}(\mathrm{clS})) \cup$ int $(\mathrm{cl}(\mathrm{int} S))$. The class of all G-open sets in $\mathrm{X}$ will be denoted by $\mathrm{GO}(\mathrm{X})$.

*Department of Mathematics, College Science for Women, University of Baghdad 
It is obvious that $\mathrm{PO}(\mathrm{X}) \cup \mathrm{SO}(\mathrm{X}) \subset_{\mathrm{BO}}$ (X) $\subset_{\mathrm{SPO}}(\mathrm{X}) \subset_{\mathrm{GO}}(\mathrm{X})$ and we shall show that the equality inclusions cannot be replaced with equalities between $\mathrm{GO}(\mathrm{X})$ and $\mathrm{PO}(\mathrm{X}), \mathrm{SO}(\mathrm{X})$ respectively.

EXAMPLE. Consider the set $\mathrm{R}$ of real numbers with the usual topology, and let $\mathrm{S}=[0,1] \cup((1,2) \cap \mathrm{Q})$ where $\mathrm{Q}$ stands for the set of rational numbers. Then $\mathrm{S}$ is $\mathrm{G}-$ open but neither semi-open nor preopen.

PROPOSITION 2.1. Let $S$ be a subset of a space $(X, T)$, if $S \subset p c l($ pint $S)$ then $S$ is G-open set.

Proof. Since $S \subset \operatorname{pcl}($ pintS) then by

Proposition1. we have:

$\mathrm{S} \subset_{\text {pint } S} \cup \mathrm{cl}($ intS) and

$S \subset$ int $(\mathrm{clS}) \cup \mathrm{cl}(\mathrm{intS})$ and so

$\mathrm{clS} \subset_{\mathrm{cl}(\mathrm{int}}(\mathrm{clS}) \cup \mathrm{cl}($ int $\mathrm{S})$ and since

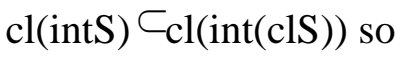

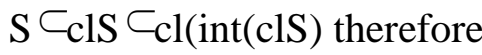

$\mathrm{S} \subset \mathrm{cl}(\mathrm{int}(\mathrm{clS})) \cup \operatorname{int}(\mathrm{cl}(\mathrm{int} S))$ so $\mathrm{S}$ is G-

open set.

PROPOSITION 2.2. . Let $S$ be a subset of a space $(X, T)$, if $S$ is $G$-open and if int $(\mathrm{cl} \mathrm{S}) \subset B \subset \mathrm{cl}(S)$ then $B$ is semiregular

Proof.since $S \subset \mathrm{cl}($ int $(\mathrm{cl} S)) \cup \operatorname{int}(\mathrm{cl}(\mathrm{int} S))$ Then $\operatorname{cl} S \subset \mathrm{cl}(\mathrm{int}(\mathrm{cl} S)) \cup \mathrm{cl}(\mathrm{int} \quad(\mathrm{cl}(\mathrm{int} S))$ so $\operatorname{cl} S \subset \mathrm{cl}(\operatorname{int}(\mathrm{cl} S))$. Therefore int (clS) $\subset \mathrm{B} \subset \mathrm{cl} S \subset \mathrm{cl}(\operatorname{int}(\mathrm{cl} S))$, then $\quad \operatorname{int}(\mathrm{cl} S) \subset$ $\mathrm{B} \subset \mathrm{cl}$ (int $(\mathrm{cl} S)$ ) and since $\operatorname{int}(\mathrm{cl} S)$ is regular open. Then B is semi-regular.

\section{On topology generated by $G$-open} sets.

Although none of $\mathrm{SO}(X)$, $\mathrm{BO}(X), \mathrm{PO}(X)$, and $\mathrm{GO}(X)$ is a topology on $X$, each these classes generates a topology in a natural way. Let $T(s)=\{V \subset$ $X \mid \quad V \cap S \in S$ whenever $S \in S\}$, where $s$ stands for $S O(X), \mathrm{PO}(X), \mathrm{GO}(X) \mathrm{SPO}(X)$ respectively. Clearly $T(s)$ is a topology on $X$ larger than $T$. The topology generated by $\operatorname{PO}(X)$ was studied in [6] and denoted by $T_{\gamma}$. The closure and the interior of a set $S$ in $\left(X, T_{\gamma}\right)$ are denoted by $\operatorname{cl}_{\gamma} S$ and int $S$, respectively. In [8] the the topology generated by $\operatorname{SPO}(X)$ was studied and we will denoted by $T_{s p o}$. In [9] the the topology generated by $\mathrm{BO}(X)$ was studied and denoted by $T_{b}$. The topology generated by $G$-open sets will be denoted by $T_{\mathrm{G}}$ and we shall prove that $\mathrm{T}_{\gamma} \subset \mathrm{T}_{\mathrm{b}} \subset \mathrm{T}_{\text {spo }} \subset \mathrm{T}_{\mathrm{G}}$. We first recollect some results.

PROPOSITION 3. If $S$ is G-open subset of a space $(X, T)$ then: $S=$ spint $S \cup$ int $_{\alpha} S$. Proof. By Proposition 1.1 we have spint $S \cup$ int $_{\alpha} S=[S \cap \operatorname{cl}(\operatorname{int}(\operatorname{cl} S)] \cup[\quad S \cap$ int $(\operatorname{cl}(\operatorname{int} S)]=S \cap[\operatorname{cl}(\operatorname{int}(\operatorname{cl} S) \cup \operatorname{int}(\operatorname{clint} S)]=S$

PROPOSITION 3.1 Let $S$ be a G-open subset of a space $(X, T)$ such that int $S=\Phi$ Then $S$ is semi-preopen.

Proof. The proof is very easy and will not be given

PROPOSITPION 3.2.[6] Let $S$ be a subset of a space $(X, T)$. Then cly $_{y}$ int $S=c l($ int $S)$.

PROPOSITPION 3.3.[8] Let $S$ be a subset of a space $(X, T)$. Then $S \in T_{\gamma}$ if and only if $S=G \cup H$ with $G \in T \alpha$ and $\{h\} \in P O(X)$ for every $h \in H$ ๘

The last result has an immediate consequence.

COROLLARY 3.4. [9] Let $v \in T_{\gamma}$ and $G \in$ $\mathrm{SO}(X)$ then $v \cap G \in \mathrm{BO}(X)$.

By previous corollary, and since $\mathrm{BO}(X) \subset \mathrm{GO}(X)$ we have the following- ng Lemma

LEMMA 3.5. Let $v \in T_{\gamma}$ and $D \in S O(X)$ then $v \cap D \in G O(X)$.

PROPOSTPION 3.6. [7] The intersection of semi-open and a preopen set is semipreopen set.

Since every semi-preopen set is G-open set. Therefore the previous Proposition tell the following Proposition.

PROPOSTPION 3.7. The intersection of semi-open and a preopen set is G-open set. 
PRROPOSITION 3.8. [7] For a space $(X, T)$ and $x \in X$ the following are equivalent:
(a) $\quad\{\mathrm{X}\} \in \mathrm{SPO}(X)$
(b) $\{\mathrm{X}\} \in \mathrm{PO}(X)$.
(c) $\{\mathrm{X}\} \in \mathrm{T}_{\gamma}$

Since every semi-preopen set is G-open set. Therefore the previous Proposition tell the following Proposition

PRROPOSITION 3.9. If $\{X\} \in G O(X)$. Then $\{X\} \in T_{\gamma}$.

Now we are in ready to prove our main result.

PRROPOSITION 3.10. Let $(X, T)$ be a space and $v \in T_{G}$. Then $S=v \backslash$ int (cllint v))is semi-preopen set.

Proof. Since int(cl(intv)) is semi-closed, then [int(cl(intv)) $]^{\mathrm{c}}$ is semi-open and so G-open set, but $\mathrm{v} \in \mathrm{T}_{\mathrm{G}}$, then vlint(cl(int $\mathrm{v}))=\mathrm{v} \cap\left[\operatorname{int}(\mathrm{cl}(\text { intv) })]^{\mathrm{c}}=S\right.$ is G-open .On other hand ,int $S=\Phi$ and so $S$ is semipreopen by proposition 3.1.

LEMMA 3.11. In any space $(X, T)$ we have: (1) [9] $T_{\gamma}=T_{b}$.

(2) [7] $T_{\gamma}=T_{s p o}$.

THEOREM 3.12. Let $(X, T)$ be a space Then $T_{\gamma} \subset T_{G}$.

Proof. Let $\mathrm{v} \in \mathrm{T}_{\gamma}$ and $S \in \mathrm{GO}(X)$, Then $S=$ spint $S \cup \operatorname{int}_{\alpha} S$ by proposition 3 . Hence $\mathrm{v} \cap S=(\mathrm{v} \cap$ spint $S) \cup\left(\mathrm{v} \cap \operatorname{int}_{\alpha} S\right)$ is $G$-open by Lemma 3.5 and so $\mathrm{v} \in \mathrm{T}_{\mathrm{G}}$.

By Theorem 3.12 and Lemma3.11, it is very easy to proof the following Corollary
COROLLARY 3.13. In any space $(X, T)$ we have $T_{\gamma} \subset T_{b}, \subset T_{s p o} \subset T_{G}$.

\section{REFERENCES:}

1.Levine, N., 1963. semi-open sets and semi-continuity in topological space, Amer. math. Monthly $70: 36-41$.

2.Corson, H.H. and Michael E., 1964. Metrizability of certain countable unions, Illinois J.Math. 8 : 351-360.

3.Andrijevic, D ., 1986. Semi-preopen sets, ibid. 38 :24-32.

4.Jancovic, D.S., 1985. On semiseparation properties, Indian J. pure Appl. Math., 16 (9): 957-964.

5.Maio, D. and Noiri T. , 1987.On $s$ closed space, Indian J. pure Appl. Math.,18 no.3: 226-233.

6.Andrijevic, D ., 1987.On the topology generated by preopen sets, ibid. 39 : 367-376.

7.Janster, M. and Andrijevic D., 1988. On some questions concerning semipreopen sets, J. Inst. Math. and Comp. Sci. (Math. Ser.) 1 : 65-75.

8.Andrijevic, D . , 1992. On SPOequivalent topologies,Suppl. Rend.Circ. Mat. Palermo 29:317-328.

9.Andrijevic, D . , 1996. On b-open sets , Mat. Vesnik,48 : 59-64.

10. Njastsd, O., 1965. On some classes of nearly open sets, Pacific J.Math. 15: 961-970.

11. Mashhour, A.S., Abd El-Monsef M.E. and El-Deeb S.N., 1982. On precontinu- -ous and weak precontinuous mappings, Proc. Math.Phys. Soc. Egypt 53: 47-53.

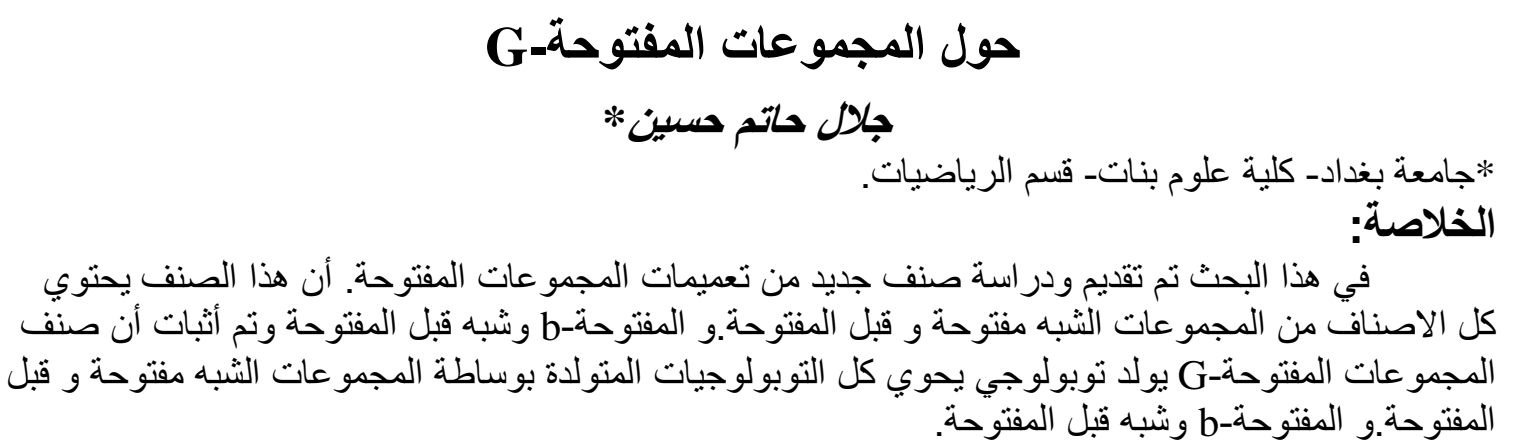

